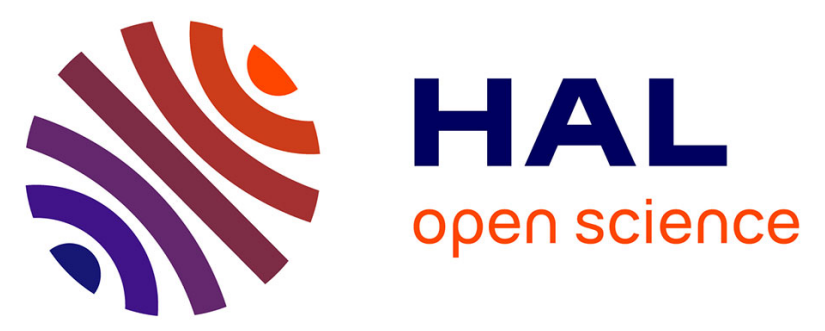

\title{
Free and Cued Selective Reminding Test - accuracy for the differential diagnosis of Alzheimer's and neurodegenerative diseases: A large-scale biomarker-characterized monocenter cohort study (Clin AD)
}

Stéphane Epelbaum, Marc Teichmann, Dalila Samri, Marcel Levy Nogueira, Harald Hampel, Foudil Lamari, Bruno Dubois, Agnès Michon

\section{To cite this version:}

Stéphane Epelbaum, Marc Teichmann, Dalila Samri, Marcel Levy Nogueira, Harald Hampel, et al.. Free and Cued Selective Reminding Test - accuracy for the differential diagnosis of Alzheimer's and neurodegenerative diseases: A large-scale biomarker-characterized monocenter cohort study (ClinAD). Alzheimer's \& Dementia: the Journal of the Alzheimer's Association, 2017, 13 (8), pp.913 - 923. 10.1016/j.jalz.2016.12.014 . hal-01672862

\section{HAL Id: hal-01672862 \\ https://hal.inria.fr/hal-01672862}

Submitted on 27 Dec 2017

HAL is a multi-disciplinary open access archive for the deposit and dissemination of scientific research documents, whether they are published or not. The documents may come from teaching and research institutions in France or abroad, or from public or private research centers.
L'archive ouverte pluridisciplinaire HAL, est destinée au dépôt et à la diffusion de documents scientifiques de niveau recherche, publiés ou non, émanant des établissements d'enseignement et de recherche français ou étrangers, des laboratoires publics ou privés. 


\title{
Free and Cued Selective Reminding Test - accuracy for the differential diagnosis of Alzheimer's and neurodegenerative diseases: a large-scale biomarker-characterized monocenter cohort study (ClinAD)
}

\author{
Marc Teichmann $^{\mathrm{a}, \mathrm{b}, *, 1}$, Stéphane Epelbaum ${ }^{\mathrm{a}, \mathrm{c}, 1}$, Dalila Samri ${ }^{\mathrm{a}, 1}$, Marcel Levy Nogueira ${ }^{\mathrm{a}, \mathrm{d}}$, \\ Agnès Michon $^{\mathrm{a}}$, Harald Hampel ${ }^{\mathrm{a}, \mathrm{e}}$, Foudil Lamari ${ }^{\mathrm{f}}$, Bruno Dubois ${ }^{\mathrm{a}, \mathrm{b}}$ \\ ${ }^{a}$ Department of Neurology, Institut de la mémoire et de la maladie d'Alzheimer, Centre de Référence 'Démences Rares', Hôpital de la Pitié-Salpêtrière, AP-HP, \\ Paris, France \\ ${ }^{b}$ Institut du Cerveau et de la Moelle Epinière (ICM), ICM-INSERM 1127, FrontLab, Paris, France \\ ${ }^{c}$ Institut du Cerveau et de la Moelle Epinière (ICM), ICM-INSERM 1127, Team Alzheimer's and Prions Diseases, Paris, France \\ ${ }^{d}$ Ecole Polytechnique, LIX, Paris-Saclay University, Palaiseau, France \\ ${ }^{e}$ AXA Research Fund and UPMC, Sorbonne Universities, Pierre and Marie Curie University, Paris 06, INSERM, CNRS, Brain and Spine Institute (ICM), Paris, \\ France \\ ${ }^{f}$ Department of Metabolic Biochemistry, Hôpital de la Pitié-Salpêtrière, AP-HP, Paris, France
}

Abstract

\begin{abstract}
Introduction: The International Working Group recommended the Free and Cued Selective Reminding Test (FCSRT) as a sensitive detector of the amnesic syndrome of the hippocampal type in typical Alzheimer's disease (AD). But does it differentiate AD from other neurodegenerative diseases?

Methods: We assessed the FCSRT and cerebrospinal fluid (CSF) AD biomarkers in 992 cases. Experts, blinded to biomarker data, attributed in 650 cases a diagnosis of typical AD, frontotemporal dementia, posterior cortical atrophy, Lewy body disease, progressive supranuclear palsy, corticobasal syndrome, primary progressive aphasias, "subjective cognitive decline," or depression.

Results: The FCSRT distinguished typical AD from all other conditions with a sensitivity of $100 \%$ and a specificity of $75 \%$. Non-AD neurodegenerative diseases with positive AD CSF biomarkers ("atypical AD") did not have lower FCSRT scores than those with negative biomarkers.

Discussion: The FCSRT is a reliable tool for diagnosing typical AD among various neurodegenerative diseases. At an individual level, however, its specificity is not absolute. Our findings also widen the spectrum of atypical AD to multiple neurodegenerative conditions.
\end{abstract}

(C) 2017 The Alzheimer's Association. Published by Elsevier Inc. All rights reserved.

\section{Introduction}

In clinical and pharmacological trials targeting Alzheimer's disease $(\mathrm{AD})$, the most prevalent neurodegenerative disease in the world [1], the use of highly specific

Disclosures: Marc Teichmann, Stéphane Epelbaum, Dalila Samri, Foudil Lamari, Marcel Levy Nogueira, Agnès Michon, Harald Hampel, and Bruno Dubois report no disclosures relevant to the manuscript.

${ }^{1}$ These authors contributed equally to the manuscript.

*Corresponding author. Tel.: +3314216 75 34; Fax: +3314216 7504.

E-mail address: marc.teichmann@psl.aphp.fr neuropsychological tests is indispensable for identifying the typical $\mathrm{AD}$ phenotype to avoid erroneous patient inclusions. Efficient neuropsychological tests would also decrease negative results of invasive second-line examinations such as lumbar puncture for cerebrospinal fluid (CSF) biomarker analyses, which might have a significant impact on the costs and the outcome of such trials. In this vein, the Free and Cued Selective Reminding Test (FCSRT) has been recommended by the International Working Group (IWG) as a reliable tool for the assessment of episodic memory failure that constitutes the core feature of typical amnesic $\mathrm{AD}[2,3]$. 
The FCSRT has been extensively studied during the past 30 years [4-10]. It evaluates the ability to learn a list of 16 written words that are presented with a semantic cue to control for memory encoding. Memory recall is then assessed by asking to retrieve the words first spontaneously ("free recall") and then with the help of a semantic cue for those items that were not retrieved ("total recall" = "free recall" + "cued recall") [6]. In contrast with most of the other memory tests the main advantages of the FCSRT are (1) that the encoding of the items is controlled by cuing to exclude "simple" attention disorders and (2) that semantic cuing facilitates the retrieval of stored information thus distinguishing between simple retrieval difficulties (facilitated by cuing), encountered, for example, in frontal dysfunction, and genuine storage deficits characterizing typical $\mathrm{AD}$ (not facilitated by cuing). Furthermore, intrusions, that is, erroneously produced items during the cued recall, suggest amnesic distortions reflecting impaired episodic memory storage. Hence, the FCSRT enables the identification of memory storage failure defining the amnesic syndrome of the hippocampal type [11], primarily characterized by insensitivity to cueing and by low total recall. Given that the amnesic syndrome of the hippocampal type has been shown to be strongly related to typical $\mathrm{AD}$ [10], even at a prodromal stage of the disease [9], the IWG proposed in 2007 to implement the FCSRT within the core diagnostic criteria of typical AD [2].

However, the aforementioned studies assessing the discriminative diagnosis value of the FCSRT suffer from several limitations. First, they were conducted in research settings exploring relatively small patient cohorts or focussing mainly on $\mathrm{AD}$ and, besides some exceptions, without including other identified neurodegenerative diseases. Second, they did not use the gold-standard core feasible CSF biomarkers to identify the underlying pathophysiology of $\mathrm{AD}$ that would have decreased the risk of false diagnoses.

In the present study, we aimed at evaluating the reliability of the FCSRT to detect typical AD in a large-scale monocentric memory-clinic cohort of patients with various neurodegenerative diseases who underwent standardized CSF biomarker assessments and who were clinically diagnosed having $\mathrm{AD}$ or eight other age-related clinically relevant neurodegenerative diseases, subjective cognitive decline (SCD) [12], or depression. CSF biomarkers were used as biological surrogate markers according to the current research criteria of Dubois et al. [3], allowing for the in vivo characterization of underlying Alzheimer's pathology. No definitive neuropathological data were available at the time of the present study.

\section{Methods}

\subsection{Patient cohort and data banking}

Patients were recruited in our tertiary memory center (Institute for Memory and AD, Pitié-Salpêtrière University
Hospital, Paris). This institute includes the national referral centers for "Young Onset AD" and for "Rare Dementias." French guidelines for the evaluation of such patients recommend a standardized neuropsychological evaluation, brain imaging, and the analysis of the AD CSF biomarker profile [13]. Our large-scale cohort of patients with typical AD and other neurocognitive diseases ("ClinAD") consists of a total of 992 patients followed at our center from 2005 to 2014 . All had extensive neurological and neuropsychological evaluations and underwent lumbar puncture for the analysis of CSF AD biomarkers. They also had neuroimaging with magnetic resonance imaging (MRI) $(\mathrm{n}=295)$, and/or with single-photon emission-computed tomography and/or ${ }^{18}$ F-2-fluoro-2-desoxy-D-glucose positron emission tomography (FDG-PET) $(\mathrm{n}=210)$. Four experienced neurologists (MT, SE, ML, and AM) reviewed the patient files, blinded to the biomarker results, and checked and validated consecutively the diagnosis for each participant. A neuropsychologist (DS) collected data of the Mini-Mental State Examination (MMSE) [14], the Frontal Assessment Battery (FAB) [15], and the FCSRT [6,16]. The amnesic syndrome of the hippocampal type, operationalized according to Sarazin et al. [9], by a free recall less than 17 of 48 or a total recall less than 40 of 48 , was searched in each participant using a computerized patient file (e-CRF) filled in prospectively and allowing for data extraction (http://en.evolucare. com/patient-file-software.html). These FCSRT cut-off values, indicating memory storage deficits, have been derived from the identification of mild cognitive impairment (MCI) subjects who declined to dementia stages of $\mathrm{AD}$ as opposed to MCI subjects who remained stable over time [9]. CSF biomarker values were entered into a second database in our biochemistry department. The two databases were then merged, anonymized, and monitored. All clinical and biological data were generated during a routine clinical work-up and were retrospectively extracted for the purpose of this study. Therefore, according to French legislation, explicit consent was waived. However, regulations concerning electronic filing were followed, and patients and their relatives were informed that anonymized data could be used in research investigations and particularly for the present study. Moreover, the local ethical committee approved this study in participants with SCD who gave their signed informed consent.

\subsection{Diagnosis procedure}

The diagnosis for typical AD was based on international consensus research criteria (prodromal and dementia stages) [3] but taking into account exclusively the clinical phenotype independently from, and blinded to, CSF biomarkers. Typical AD patients had an amnesic syndrome of the hippocampal type, associated or not with nonpredominant symptoms of aphasia, apraxia, agnosia, or executive disorders. The identification of the amnesic syndrome of the hippocampal type was based on the application of the FCSRT 
that contained the following test stages: (1) learning a list of 16 written words presented with a semantic cue to control for memory encoding; (2) assessing memory recall by asking to retrieve the 16 words first spontaneously (free recall) and then with the help of a semantic cue for those items that were not spontaneously retrieved (total recall $=$ free recall + cued recall); (3) repeating the procedure three times to provide a free recall and a total recall score of 48 ; (4) calculating the sensitivity to cueing by the formula (["sum of the 3 total recalls" - "sum of the 3 free recalls"]/[48 "sum of the 3 free recalls"]); (5) reapplying the same test procedure 30 minutes later to evaluate the free delayed recall and the total delayed recall; and (6) exploring the ability to recognize the tested items (seen versus not seen).

International diagnostic criteria were also applied to identify the clinical phenotype of eight other neurodegenerative diseases of the study cohort: frontotemporal dementia of the behavioral type (bv-FTD) [17], primary progressive aphasia (PPA) of the logopenic (lv-PPA), semantic (sv-PPA), or nonfluent/agrammatic (nfv-PPA) type [18], corticobasal syndrome (CBS) [19], progressive supranuclear palsy (PSP) [20], posterior cortical atrophy (PCA) [21], and Lewy body disease (LBD) [22]. International diagnostic criteria were used to identify SCD [12] and depression [23]. More specifically, regarding depression, we applied the Montgomery-Assberg Depression Rating Scale (MADRS) [24] to quantify and to screen for major depressive syndromes (MADRS $\geq 20$ ), according to the Diagnostic and Statistical Manual of Mental Disorders (Fifth Edition) [23]. Major depression was also confirmed by an extensive psychological examination. In the "depression group" of the study there were no patients with degenerative diseases or lesion-related depression as reflected by normal MRI, FDG-PET, and/or CSF biomarkers and the absence of cognitive decline during follow-up. Furthermore, in the groups of neurodegenerative diseases, the rate of major depression was low ( $<10 \%$ according to the MADRS), and the proportion of such depressed patients was equivalent in these groups that allowed for avoiding intergroup biases concerning the interpretation of abnormal FCSRT scores.

The FCSRT, MMSE, and FAB were applied to all participants. Patients with significant vascular brain lesions or mixed diseases were excluded from further analyses to avoid mixtures of vascular and degenerative processes. More specifically, we used the MRI staging of Fazekas et al. [25] to exclude patients with significant lesions of vascular origin (Fazekas score $>2$ ), which might interfere with cognitive/ memory functioning and, therefore, generate biases regarding FCSRT results. Only slight lesions of leucoaraiosis were accepted for inclusion in the study. Furthermore, given that MRI white matter hyperintensities in neurodegenerative diseases are not necessarily vascular lesions, but might reflect the degenerative process itself, we did not exclude patients with hyperintensities that were not obviously of vascular origin such as juxtacortical/periventricular patterns next to atrophied cortical regions [26].

A subsample of 30 patients was diagnosed by each of the four expert neurologists to calculate interrater reliability coefficients.

\subsection{CSF biomarkers}

CSF analyses were performed at the clinical biochemistry department of the Pitié-Salpêtrière University Hospital, including the quantification of total tau protein (t-tau), tau

\section{Diagnostic distribution $\mathrm{N}=650$}

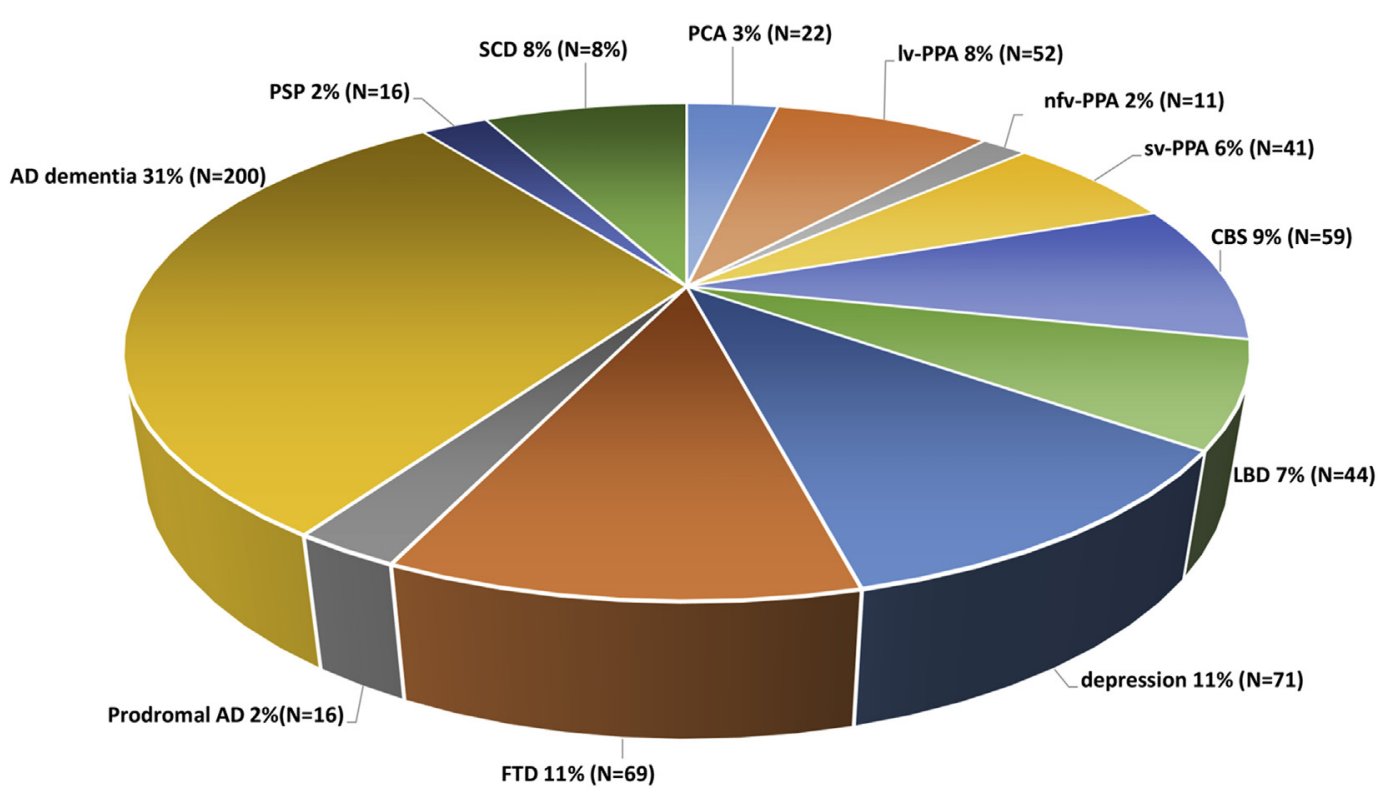

Fig. 1. Diagnostic distribution of typical Alzheimer's disease (AD), the eight other neurodegenerative diseases, subjective cognitive decline (SCD), and depression. 
Table 1

Demographic, neuropsychological, and biomarker characteristics by diagnosis and comparison between typical AD, eight other neurodegenerative diseases, $\mathrm{SCD}$, and depression

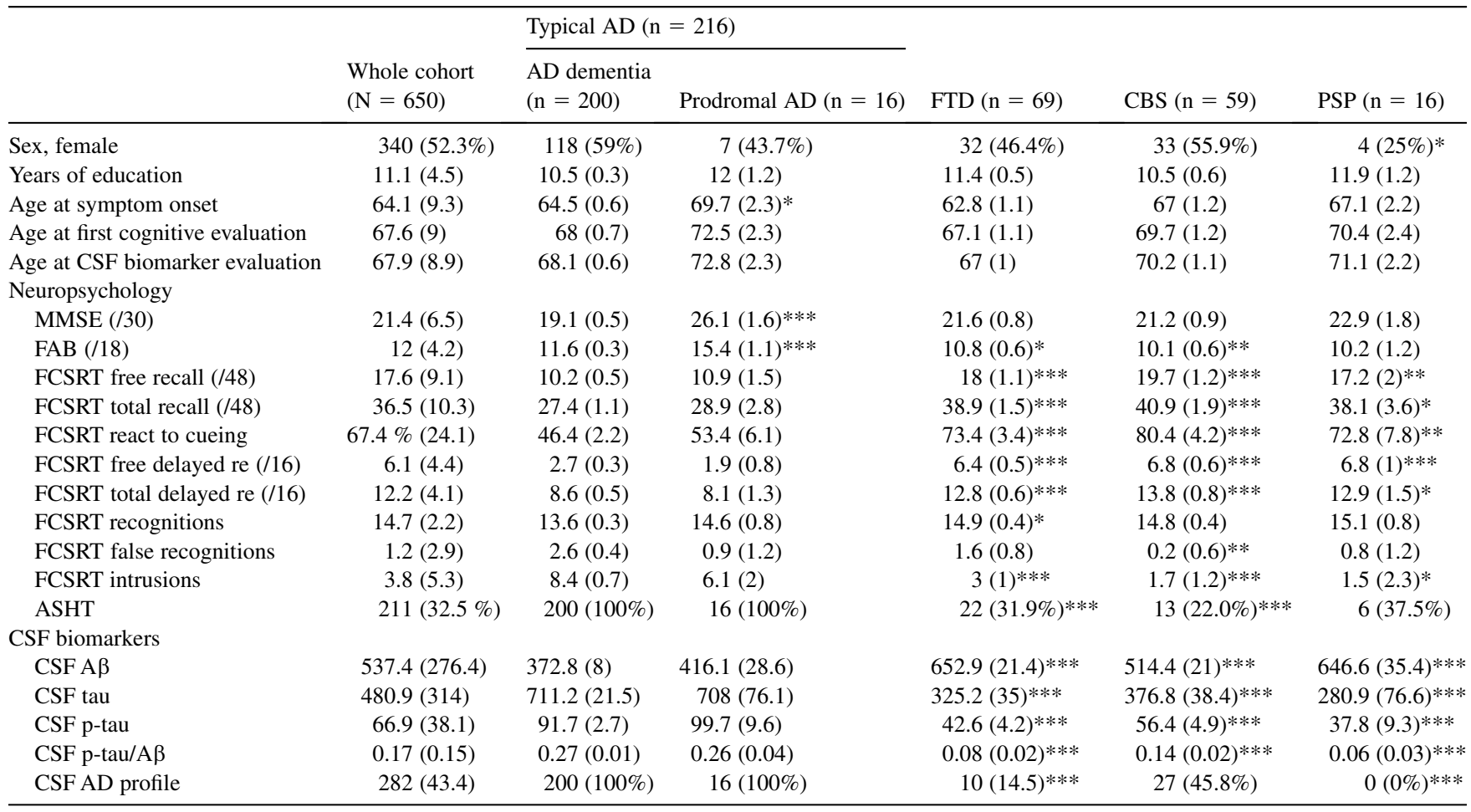

Abbreviations: $\mathrm{A} \beta$, amyloid $\beta$; AD, Alzheimer's disease; ASHT, amnesic syndrome of the hippocampal type; CBS, corticobasal syndrome; CSF, cerebrospinal fluid; FAB, Frontal Assessment Battery; FCSRT, Free and Cued Selective Reminding Test; FTD, frontotemporal dementia; free delayed re, free delayed recall; LBD, Lewy body disease; lv-PPA, logopenic primary progressive aphasia; MMSE, Mini-Mental State Examination; nfv-PPA, nonfluent/agrammatic primary progressive aphasia; PCA, posterior cortical atrophy; PSP, progressive supranuclear palsy; p-tau, phosphorylated tau; react to cueing, reactivity to cueing; SCD, subjective cognitive decline; sv-PPA, semantic primary progressive aphasia; total delayed re, total delayed recall.

NOTE. In brackets: standard deviations or \%. The two "Typical AD" columns = comparison between AD dementia and prodromal AD. All subsequent columns $=$ comparison between $\mathrm{AD}$ and the other neurodegenerative conditions, depression, and $\mathrm{SCD} . * P<.05, * * P<.01, * * * P<.001$.

protein phosphorylated at threonine $181\left(\mathrm{p}-\operatorname{tau}_{181}\right)$, and amyloid- $\beta$ 1-42 (A $\left.\beta_{1-42}\right)$ peptide. CSF samples were centrifuged for $10 \mathrm{~min}$ at $3500 \mathrm{rpm}$ at $4{ }^{\circ} \mathrm{C}$ to remove cells, aliquoted to $0.4 \mathrm{~mL}$ samples in polypropylene tubes, and then stored at $-80^{\circ} \mathrm{C}$ until analysis. Biomarker concentrations of t-tau, $\mathrm{p}$-tau ${ }_{181}$, and $\mathrm{A} \beta_{1-42}$ were analyzed in duplicate using the double antibody sandwich ELISA method (Fujirebio). We also calculated derived ratios from single biomarkers including t-tau/A $\beta_{1-42}$ and $p$-tau ${ }_{181} / A \beta_{1-42}$ ratios. The ratio cut-off indicative of $\mathrm{AD}$ was set at $\mathrm{p}-\mathrm{tau}_{181} /$ $\mathrm{A} \beta_{1-42}>0.11$ based on studies with postmortem verification of $\mathrm{AD}$ diagnosis [27,28] and on a large longitudinal monocentric cohort [29]. This stringent approach was used to provide robust cut-offs validated by neuropathological examinations, even if some authors consider that individual biomarker abnormalities of, for example, $\mathrm{A} \beta_{1-42}$ might be sufficient to identify AD [30].

\subsection{Statistical analyses}

T-tests were performed between prodromal and dementia stage $\mathrm{AD}$ and then between all $\mathrm{AD}$ patients and the other groups. Statistical significance was considered when $P$ was inferior to .05 . We then dichotomized the non-AD groups based on the CSF profile indicative, or not, of underlying Alzheimer's pathology to identify atypical variants of $\mathrm{AD}$ in all the eight neurodegenerative conditions and to explore whether underlying Alzheimer's pathology as such might affect memory scores of the FCSRT. Furthermore, we conducted correlation analyses using linear regressions to identify whether CSF biomarker values ( $\mathrm{p}$-tau ${ }_{181} / \mathrm{A} \beta_{1-42}$ ratio) have an relationship with FCSRT scores in the AD group, in the entire group of the eight other neurodegenerative conditions, and in the whole group of degenerative diseases (AD plus the eight other neurodegenerative conditions). All statistical analyses were performed using the jmp software (SAS, 2007).

\section{Results}

\subsection{Patient cohort (ClinAD)}

Among the 992 cases, 342 were excluded from the analyses because of significant vascular disease on MRI or because of mixed disease patterns. The remaining 650 
Table 1

Continued.

\begin{tabular}{|c|c|c|c|c|c|c|}
\hline $\operatorname{LBD}(\mathrm{n}=44)$ & $\operatorname{PCA}(n=22)$ & Sv-PPA $(n=41)$ & Lv-PPA $(\mathrm{n}=52)$ & Nfv-PPA $(n=11)$ & Depression $(\mathrm{n}=71)$ & $\operatorname{SCD}(n=49)$ \\
\hline $11.7(0.7)$ & $11.3(1)$ & $11.9(0.7)$ & $11.6(0.6)$ & $9.8(1.3)$ & $10.5(0.5)$ & $12.6(0.7)^{* *}$ \\
\hline $67.7(1.3)$ & $59(1.9)^{* *}$ & $62.6(1.4)$ & $64.7(1.2)$ & $66.4(2.7)$ & $58.4(1.1)^{* * *}$ & $66.4(1.5)$ \\
\hline $70.7(1.3)$ & $62.3(1.9)^{* *}$ & $66.4(1.5)$ & $68.2(1.3)$ & $70.6(2.7)$ & $62.1(1.1)^{* * *}$ & $69.6(1.6)$ \\
\hline $20.9(1)$ & $18.2(1.3)$ & $21(1)$ & $17.7(1)^{*}$ & $22.6(1.9)$ & $25.6(0.7)^{* * *}$ & $28.4(0.9)^{* * *}$ \\
\hline $11.6(0.7)$ & $10.2(1)$ & $12(0.7)$ & $10.2(0.6)^{* *}$ & $11.9(1.2)$ & $14.2(0.5)^{* * *}$ & $16.4(0.6)^{* * *}$ \\
\hline $15.2(1.2)^{* *}$ & $18.6(1.7)^{* * *}$ & $15.3(1.8)^{*}$ & $20.4(1.8)^{* * *}$ & $22.9(2.4)^{* * *}$ & $21.5(0.9) * * *$ & $29.6(1.1)^{* * *}$ \\
\hline $36.2(2.1)^{* *}$ & $41.3(3)^{* * *}$ & $32.6(3.1)^{*}$ & $43.4(3)^{* * *}$ & $43(4)^{* *}$ & $40(1.3)^{* * *}$ & $45.8(1.3)^{* * *}$ \\
\hline $66.4(4.5)^{* *}$ & $78.3(6.5)^{* * *}$ & $56 \%(6.8)$ & $85.6(6.5)^{* * *}$ & $85.9(8.5)^{* * *}$ & $72.6(2.8)^{* * *}$ & $88.7(3) * * *$ \\
\hline $4.1(0.6)$ & $7.4(0.9)^{* * *}$ & $6.4(0.9)^{* * *}$ & $9(0.8)^{* * * *}$ & $8.9(1.1)^{* * *}$ & $7.6(0.4)^{* * *}$ & $10.8(0.6)^{* * *}$ \\
\hline $18(40.9 \%)$ & $5(22.7)^{* *}$ & $9(22 \%)$ & $3(5.8 \%)^{* * *}$ & $3(27.3 \%)^{* *}$ & $26(36.6 \%)^{* * *}$ & $0(0 \%)^{* * *}$ \\
\hline $461(23.1)^{* *}$ & $393.4(30.1)$ & $605.4(26)^{* * *}$ & $437.4(21.9)^{*}$ & $659.6(47.6)^{* * *}$ & $817.7(22.2)^{* * *}$ & $827.1(31.7)^{* * *}$ \\
\hline $282(44.1)^{* * *}$ & $516.4(65.9)^{*}$ & $396.2(46.4)^{* * *}$ & $650.4(44.5)$ & $370.7(92.5)^{* * *}$ & $255.7(33.5)^{* * *}$ & $281.8(35.9)^{* * *}$ \\
\hline $46.3(5.5)^{* * *}$ & $71.2(8.1)^{*}$ & $59(6.1)^{* * *}$ & $83.7(5.4)$ & $57.3(11.5)^{* *}$ & $46.1(4.1)^{* * *}$ & $48.5(4.6)^{* * *}$ \\
\hline $0.12(0.02)^{* * *}$ & $0.2(0.03)$ & $0.14(0.02)^{* * * *}$ & $0.24(0.02)$ & $0.12(0.04)^{* *}$ & $0.06(0.01)^{* * *}$ & $0.07(0.02)^{* * *}$ \\
\hline $22(50 \%) * * *$ & $18(81.8 \%)$ & $15(36.6 \%)$ & $41(78.8 \%)^{* *}$ & $5(45.4)^{* * *}$ & $7(9.9 \%) * * *$ & $7(14.3 \%)^{* * *}$ \\
\hline
\end{tabular}

patients fit one of the aforementioned diagnostic categories: typical $\mathrm{AD}(\mathrm{n}=216$, dementia stage $\mathrm{n}=200$, prodromal stage $\mathrm{n}=16)$, bv-FTD $(\mathrm{n}=69)$, PPA $(\mathrm{n}=104$, lv-PPA $\mathrm{n}=52$, sv-PPA $\mathrm{n}=41, \mathrm{nfv}-\mathrm{PPA} \mathrm{n}=11)$, CBS $(\mathrm{n}=59)$, PSP $(\mathrm{n}=16)$, PCA $(\mathrm{n}=22)$, LBD $(\mathrm{n}=44)$, SCD $(n=49)$, and depression $(n=71)$. In the subsample of 30 patients diagnosed by each of the 4 expert neurologists, the interrater reliability assessment coefficients were $>0.9$. It should be noted that the sample size of the eight non$\mathrm{AD}$ conditions is smaller than the size of the typical AD group reflecting lower prevalence of these eight neurodegenerative conditions. The sample sizes are, however, not negligible thus allowing for statistically informative results. The distribution of the different diagnostic groups is represented in Fig. 1.

\subsection{CSF biomarkers}

Of the 650 subjects, 368 (56.6\%) exhibited a CSF profile indicative of underlying Alzheimer's pathology. Among these, 200 of 200 were classified in the AD dementia group (100\%), 16 of 16 in the prodromal AD group (100\%), 138 of
$314(43.9 \%)$ in the other neurodegenerative diseases, and 14 of 120 in the depression and SCD group (11.7\%). Among the other neurodegenerative diseases, the PCA and the lv-PPA groups contained $81.8 \%$ and $78.8 \%$ cases with positive AD biomarker profiles, respectively. Positive CSF biomarkers were also found in LBD (50\%), CBS (45.8\%), nfv-PPA (45.4\%), sv-PPA (36.6\%), and bv-FTD (14.5\%). An AD biomarker profile had, therefore, and by definition, a sensitivity of $100 \%$ but a rather low specificity (56.1\%) for identifying typical AD of the amnesic form among all the other neurodegenerative diseases. Patients with positive or negative CSF biomarkers did not differ in terms of age, sex, or educational level. Demographic and biomarker data are summarized in Table 1.

\subsection{Free and Cued Selective Reminding Test}

In the $\mathrm{AD}$ group (dementia and prodromal stage), FCSRT scores were abnormal as compared with normative data [9], whereas the MMSE and the FAB were only impaired at the dementia stage. Abnormal FCSRT scores, that is, free recall less than 17 of 48 or total recall less than 40 of 48 , had by 


\section{MMSE}

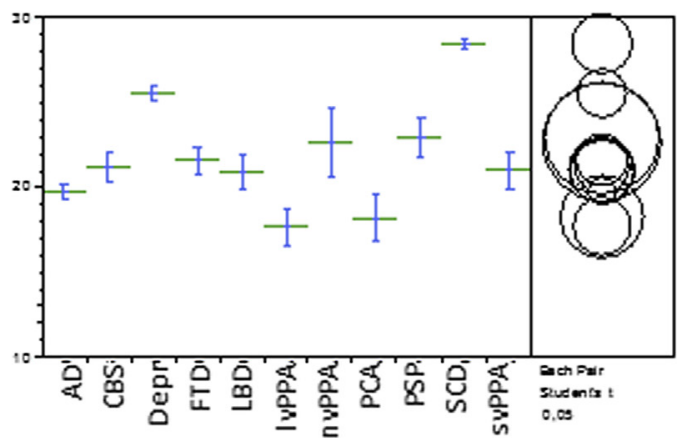

FCSRT Free recall

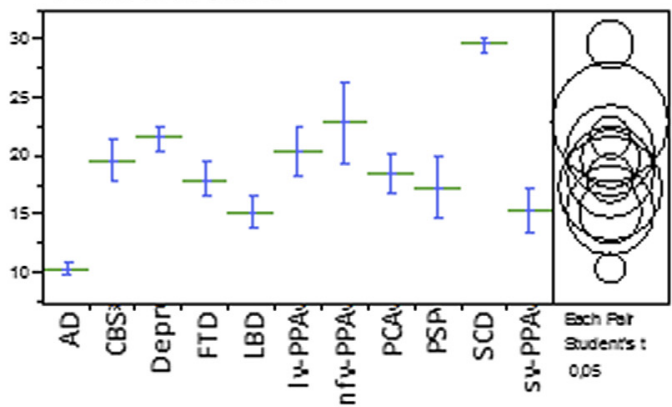

FCSRT Reactivity to cueing

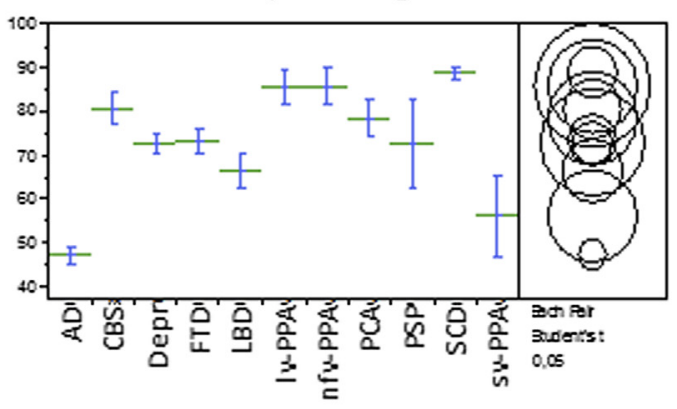

FCSRT free delayed recall

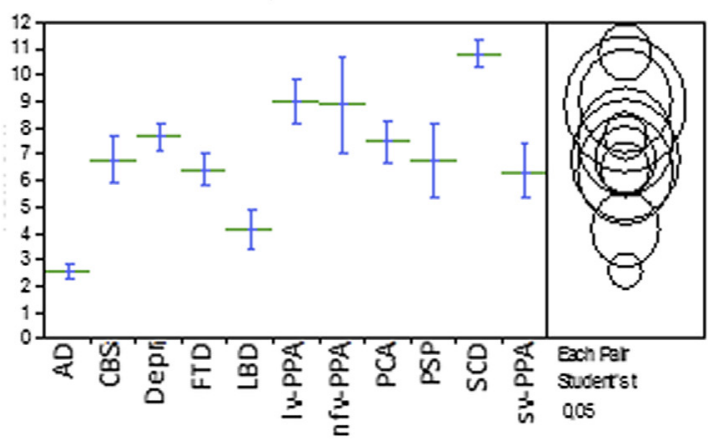

FAB

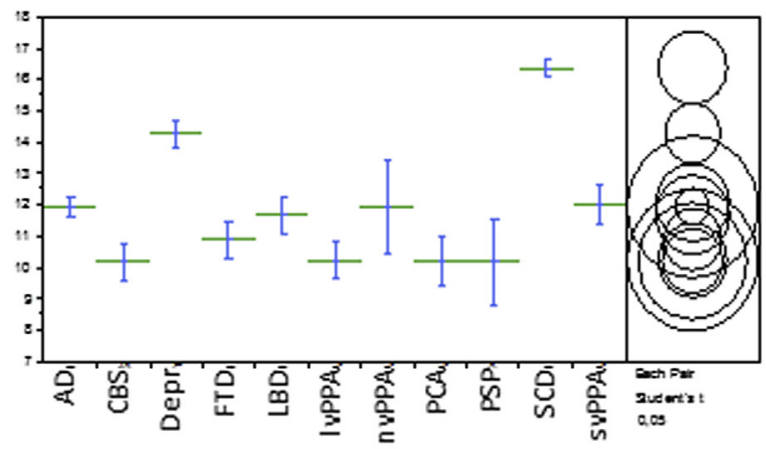

FCSRT total recall

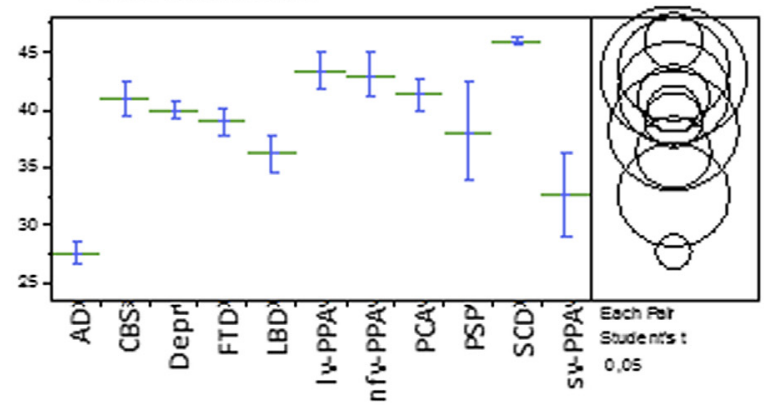

FCSRT intrusions

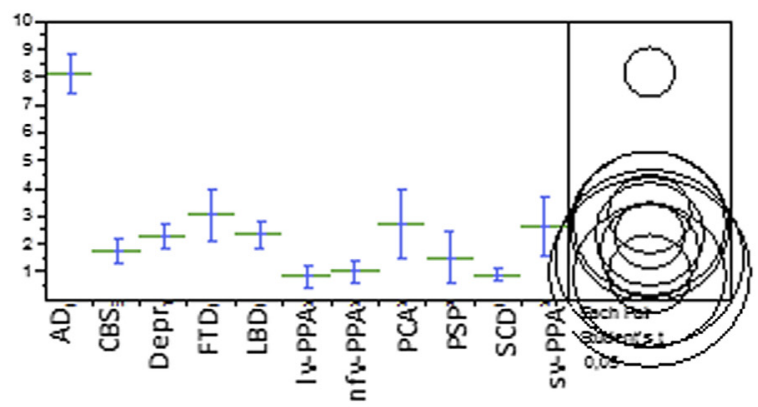

FCSRT total delayed recall

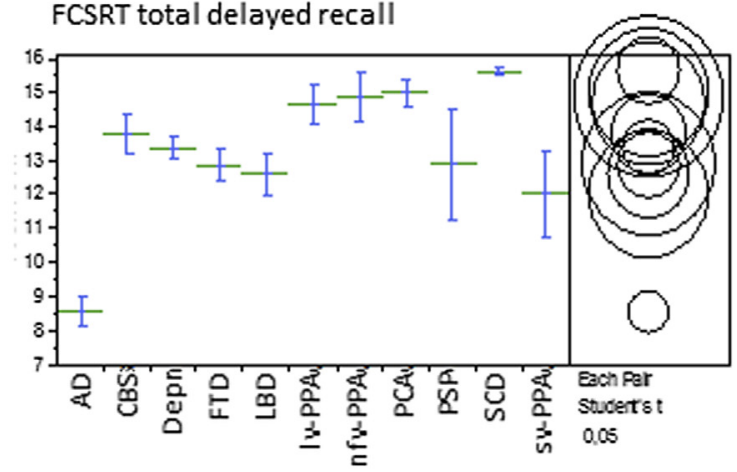

Fig. 2. Neuropsychological scores per diagnosis group (means, standard deviations) and each pair Student's $t$-test representation. Note the clear separation of Alzheimer's disease (AD) from all other diagnosis groups for the Free and Cued Selective Reminding Test (FCSRT) total delayed recall and the number of intrusions.

definition a sensitivity of $100 \%$, but a lower specificity of $74.8 \%$, to identify typical $\mathrm{AD}$, at dementia and prodromal stages, among all other degenerative diseases. MMSE and FAB scores were similar in AD dementia and in all the other neurodegenerative diseases. The delayed total recall and the number of intrusions during the total recall of the FCSRT were significantly more impaired in typical AD than in the other groups, reflecting poorer performance in long-term verbal memory and the presence of amnesic distortions specific of typical AD (see Table 1 and Fig. 2). The best 
Table 2

Comparisons between groups with positive AD biomarkers $(+)$ and negative AD biomarkers $(-)$

\begin{tabular}{|c|c|c|c|c|c|c|c|c|c|c|c|c|c|c|c|c|}
\hline & $\begin{array}{l}\text { Lv- } \\
\mathrm{PPA}+ \\
(\mathrm{n}=41)\end{array}$ & $\begin{array}{l}\mathrm{Lv}- \\
\mathrm{PPA}- \\
(\mathrm{n}=11)\end{array}$ & $\begin{array}{l}\text { Sv- } \\
\text { PPA+ } \\
(\mathrm{n}=15)\end{array}$ & $\begin{array}{l}\text { Sv- } \\
\text { PPA- } \\
(\mathrm{n}=26)\end{array}$ & $\begin{array}{l}\text { Nfv- } \\
\text { PPA+ } \\
(n=5)\end{array}$ & $\begin{array}{l}\text { Nfv- } \\
\text { PPA- } \\
(\mathrm{n}=6)\end{array}$ & $\begin{array}{l}\text { PCA }+ \\
(\mathrm{n}=18)\end{array}$ & $\begin{array}{l}\mathrm{PCA}- \\
(\mathrm{n}=4)\end{array}$ & $\begin{array}{l}\text { CBS }+ \\
(n=27)\end{array}$ & $\begin{array}{l}\text { CBS- } \\
(\mathrm{n}=32)\end{array}$ & $\begin{array}{l}\text { FTD }+ \\
(\mathrm{n}=10)\end{array}$ & $\begin{array}{l}\text { FTD- } \\
(\mathrm{n}=59)\end{array}$ & $\begin{array}{l}\mathrm{LBD}+ \\
(\mathrm{n}=22)\end{array}$ & $\begin{array}{l}\text { LBD- } \\
(\mathrm{n}=22)\end{array}$ & $\begin{array}{l}\text { SCD } \\
\text { and } \\
\text { depr }+ \\
(n=14)\end{array}$ & $\begin{array}{l}\text { SCD } \\
\text { and } \\
\text { depr- } \\
(\mathrm{n}=106)\end{array}$ \\
\hline $\begin{array}{l}\text { Age at first cognitive } \\
\text { evaluation }\end{array}$ & $68.8(1.1)$ & $65.8(2.1)$ & $68(2.6)$ & $65.5(1.9)$ & $70.4(4.4)$ & $70.8(4)$ & $61.7(1.6)$ & $65.2(3.4)$ & 70.4 (1.4) & $69(1.4)$ & $67.3(1.2)$ & $64.7(3)$ & 69.5 (1.6) & $71.8(1.5)$ & $70.3(3.1)$ & $63.6(1.1)^{*}$ \\
\hline $\begin{array}{l}\text { Age at CSF } \\
\text { biomarker } \\
\text { evaluation }\end{array}$ & $69.4(2)$ & $66.4(1)$ & $67.7(2.3)$ & $65.6(1.8)$ & $72(4.6)$ & $70.5(4.2)$ & $62.4(1.6)$ & $64.2(3.5)$ & $70.9(1.4)$ & $69.7(1.3)$ & $67.4(1.2)$ & $65.3(3.1)$ & $70.8(1.5)$ & $71.8(1.5)$ & $60.2(1.2)$ & $67.1(3.3)$ \\
\hline \multicolumn{17}{|l|}{ Neuro psychology } \\
\hline MMSE & $16.1(1.1)$ & $23(2) *$ & $22.3(1.3)$ & $18.3(1.9)$ & $24.6(3)$ & $21(2.7)$ & $17(1.4)$ & $24(3)^{*}$ & $21.4(1.3)$ & $21(1.3)$ & $14.8(1.8)$ & $22.9(0.8)^{* * *}$ & $19.2(1.2)$ & $22.9(1.3)^{*}$ & $26.7(0.4)$ & $27.1(1)$ \\
\hline FAB & $11(1.3)$ & $10(0.7)$ & $9.9(1)$ & $13.1(0.7)^{*}$ & $14.6(2)$ & $9.7(1.8)$ & $11(1.9)$ & $10(0.9)$ & $9.9(0.8)$ & $10.4(0.8)$ & $6.1(1.4)$ & $11.7(0.6)^{* * *}$ & $11.9(0.8)$ & $11.3(0.8)$ & $15.1(0.3)$ & $15(0.9)$ \\
\hline $\begin{array}{l}\text { FCSRT free recall } \\
\quad(/ 48)\end{array}$ & $20.5(3.2)$ & $20.3(3.5)$ & $16.8(3)$ & $14.3(2.5)$ & $23.7(5.9)$ & $22.2(5.1)$ & $19.6(1.9)$ & $15.7(3.3)$ & $19.5(2.5)$ & $19.8(2.5)$ & $17.9(1.5)$ & $19.3(5.5)$ & $17.6(1.9)$ & $12.5(2)$ & $24.6(0.8)$ & $26.9(2.2)$ \\
\hline $\begin{array}{l}\text { FCSRT total } \\
\text { recall (/48) }\end{array}$ & $43.3(2.3)$ & $43.5(2.3) 3$ & $35.8(5.7)$ & $30.3(4.8)$ & $41(3)$ & $44.5(2.6)$ & $41.1(1.7)$ & $42(2.9)$ & $39.5(2.1)$ & $42.2(2.1)$ & $38.8(1.2)$ & $40.7(4.4)$ & $31.9(1.9)$ & $40.1(1.8)^{* *}$ & $42.2(0.6)$ & $44.3(1.6)$ \\
\hline $\begin{array}{l}\text { FCSRT } \\
\text { reactivity to } \\
\text { cueing }\end{array}$ & $84.5(5.9)$ & $86.6(5.9)$ & $63.4(14.6)$ & $50.7(12.4)$ & $81.7(6.5)$ & $89(5.6)$ & $77.3(5.3)$ & $81.3(9.2)$ & $76.2(5.2)$ & $84.6(5.2)$ & $73.4(3)$ & $73(10.8)$ & $58.3(4.8)$ & $75.6(4.6)^{* *}$ & $78.6(1.6)$ & 84.8 (1.9) \\
\hline $\begin{array}{l}\text { FCSRT free } \\
\text { delayed } \\
\text { recall }(/ 16)\end{array}$ & $9.3(1.3)$ & $8.7(1.3)$ & $8.8(1.2)$ & $4.3(1.1)$ & $9.3(3)$ & $8.5(2.6)$ & $7.1(1)$ & $8.3(1.6)$ & $5.7(1.2)$ & $7.8(1.2)$ & $6.3(0.7)$ & $8.3(2.4)$ & $1.8(0.8)$ & $6.2(0.8)^{* * *}$ & $8.8(0.4)$ & $10.8(0.2)$ \\
\hline
\end{tabular}

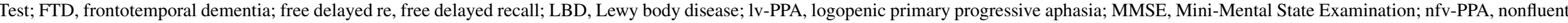

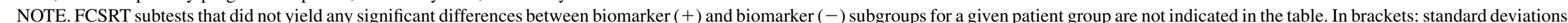
$* P<.05, * * P<.01, * * * P<.001$.
} 
discriminating threshold was found at four intrusions, with a sensitivity of $83 \%$ and a specificity of $72 \%$ to diagnose typical $\mathrm{AD}$ versus all the other patient groups of the study. However, an amnesic syndrome of the hippocampal type was also present in 105 of $385(27.3 \%)$ of patients with a diagnosis other than AD including 79 of 314 (25.2\%) of patients with other neurodegenerative diseases and 26 of 71 $(36.6 \%)$ of patients with depression. More specifically, FCSRT scores indicative of an amnesic syndrome of the hippocampal type were found in patients with LBD (40.9\%), PSP (37.5\%), bv-FTD (31.9\%), nfv-PPA (27.3\%), PCA (22.7\%), sv-PPA (22\%), CBS (22\%), and lv-PPA (5.8\%). However, normal FCSRT scores had a relatively acceptable specificity to screen out these diseases in populations that would be designated to detect typical AD (LBD [59.1\%], PSP [62.5\%], bv-FTD [68.1\%], nfv-PPA [72.7\%], PCA [77.3\%], sv-PPA [78.1\%], CBS [78.0\%], and lv-PPA [94.2\%]).

In these neurodegenerative diseases, the biomarker status did not influence the presence or absence of an amnesic syndrome of the hippocampal type, except for the LBD group who had poorer FCSRT scores when AD biomarkers were positive. Conversely, biomarker-positive patients, compared with biomarker-negative subjects, performed worse on the MMSE and/or the FAB. Results are summarized in Table 2. Regarding correlation analyses, they showed that higher biomarker ratios $\left(\mathrm{p}-\mathrm{tau}_{181} / \mathrm{A} \beta_{1-42}\right)$ indicative of underlying $\mathrm{AD}$ pathology were related to poorer free recall and total recall scores and to more intrusions on the FCSRT (all $P \mathrm{~s}<.001$ ) when considering the whole group of degenerative diseases (AD plus the eight other degenerative conditions). However, no significant correlations (all $P \mathrm{~s}>.1$ ) were found for the $\mathrm{AD}$ group alone or for the entire group of the eight other degenerative conditions.

\section{Discussion}

The present study is, to our knowledge, the first to assess the reliability of the FCSRT for detecting typical $\mathrm{AD}$ in a large-scale monocenter cohort applying the clinical phenotype $\mathrm{AD}$ diagnostic categorization recommended by the advanced IWG-2 criteria [3] and applying stringently the most recent diagnostic criteria for eight other clinically relevant age-related neurodegenerative diseases. All previous studies using this memory test were conducted in research settings to distinguish merely prodromal $A D$ from stable MCI or SCD [31] or in small-cohort investigations with highly selected patient groups without any biomarker information [5,6]. The only exception is the investigation of Wagner et al. [32] using CSF biomarkers in $185 \mathrm{MCI}$ subjects, yet without including other identified neurodegenerative diseases. Addressing these limitations, our study provides a robust validation of the reliability of the FCSRT to identify typical AD among populations of patients with various neurodegenerative diseases.
Compared with studies on MCI or SCD, in which the free recall and total recall were the most useful scores to distinguish typical AD from stable MCI or SCD [4,9,31], our findings show that all subscores of the test are sensitive to typical AD whatever its stage, but that the "delayed total recall" and the "number of intrusions" are the most discriminative indicators. These results are in line with the previous studies suggesting that intrusions depend critically on the hypometabolism of hippocampal regions, but that prefrontal regions, also damaged in more advanced $\mathrm{AD}$, might play an additional role [33]. They also reinforce and validate the diagnostic value of the FCSRT in clinical practice. Furthermore, the fact that there is no difference in the magnitude of the impairment in its subscores between $\mathrm{AD}$ dementia and prodromal $\mathrm{AD}$ provides evidence for its usefulness in assessing and detecting predementia stages of AD. Such stages correspond to an earlier phase of the disease process and, therefore, to an optimal therapeutic time window.

Low scores of free recall and total recall were also found in various other neurodegenerative diseases and in depression. In neurodegenerative diseases such abnormal scores are probably related to severe cognitive changes, including language or profound executive dysfunction, which might interfere with the performance on the FCSRT and be wrongly interpreted as episodic amnesia of the hippocampal type. However, abnormal FCSRT scores might also result from genuine damage to the hippocampi, which has been evidenced in several neurodegenerative diseases such as bv-FTD, LBD, PSP, and sv-PPA [34-37]. The concept of the amnesic syndrome of the hippocampal type should, therefore, be used cautiously suggesting that abnormal FCSRT scores do not have an absolute specificity for typical AD diagnosis. The sensitivity of $100 \%$ not only makes the FCSRT an excellent test for detecting typical AD patients but also provides some false positive results (specificity 75\%) concerning other neurodegenerative diseases. This limited specificity for typical $A D$ is a limitation of the test indicating the necessity to take into account the IWG criteria positing that typical $A D$ is a clinicobiological entity $[2,3]$, the identification of which depends on both a noninvasive clinical criterion (abnormal FCSRT scores) and a biological criterion such as positive AD CSF biomarkers or amyloiddetecting PET. One should, however, be aware that our study also shows that there are several non-AD degenerative diseases that have positive CSF biomarkers. This latter finding represents a challenge for the development of new memory tests with a similar sensitivity as the FCSRT but still better specificity for typical AD. It should also be noted that the exclusive application of biological markers is not sufficient because they do not provide the syndromic information that clinically defines a given disease such as typical amnesic AD. Thus, memory tests such as the FCSRT remain indispensable, non-invasive, 
inexpensive, and easy-to-obtain first-line tools that screen for candidates for therapeutic trials in typical AD and that provide important end points evaluating potential trial efficiency.

Despite no absolute specificity of the FCSRT for typical amnesic $\mathrm{AD}$, we advocate for continuing to use the term "amnesic syndrome of the hippocampal type" as reflected by FCSRT scores bearing in mind that it is mostly evocative of typical AD when (1) it is central or even isolated (criteria for other neurodegenerative conditions unfulfilled, for example, no socio-emotional changes suggestive of bvFTD, no extrapyramidal symptoms as in LBD, CBS, PSP); (2) it is most severe; and (3) when it is associated with other signs of hippocampal dysfunction such as intrusions [38].

Our study also confirms, enriches, and opens up the atypical AD spectrum proposed by the IWG [3] that included PCA, lv-PPA, and the so-called "frontal variant" suggestive of bv-FTD. In line with this view, PCA and lv-PPA are in our investigation among the clinical phenotypes that are most frequently associated with positive AD biomarkers $(80 \%)$. On the other hand, the prevalence of the frontal variant of $\mathrm{AD}$ is among the rarest of all syndromes with an $\mathrm{AD}$ biomarker profile in our study (14.5\%). Our findings also highlight that several other neurodegenerative entities can be associated with an $\mathrm{AD}$ biomarker profile: LBD, which could be considered as mixed AD/LBD pathology as shown by neuropathological data [39], but also CBS that was the fourth most prevalent phenotype associated with an AD biomarker profile. Similarly, nfv-PPA and sv-PPA can be associated with Alzheimer's pathology and thus, in some cases, constitute atypical variants of $\mathrm{AD}$. Interestingly, positive CSF AD biomarkers in these diseases are not related to lower FCSRT scores and numerical CSF biomarker ratios do not correlate with lower FCSRT scores in these diseases or in the typical AD group. These latter results can be explained by the fact that, in contrast with memory tests, the longitudinal changes in CSF biomarkers are not correlated with disease progression [40]. They also suggest that the brain lesion load of p-tau and $A \beta$ might not be a reliable predictor of memory deficits in neurodegenerative diseases.

In summary, based on the paradigm of the IWG considering that $\mathrm{AD}$ is a clinicobiological entity using in vivo biomarkers $[2,3]$, our data open-up the spectrum of atypical $\mathrm{AD}$ variants including biomarker-positive forms of PCA, the three main PPA variants, CBS, and bv-FTD. Depending on the syndrome, underlying AD pathology can be considered either a co-occurring pathology (e.g., for LBD) or as the main pathology. The fact that in our cohort, the prevalence of an AD-positive biomarker profile in other neurodegenerative diseases matches the prevalence described in clinicopathological cohorts $[21,41]$ suggests that the rate of false positive is low. This outcome is probably linked to the fact that the combined $\mathrm{p}$-tau ${ }_{181} / \mathrm{A} \beta_{1-42}$ cut-off chosen to consider a positive $\mathrm{AD}$ biomarker profile is stringently derived from clinicopathological and large clinical cohorts [2729]. The clinical differential diagnostic value of the AD CSF biomarker profile has been recently demonstrated in a large-scale multicenter memory clinic-based cohort study [42]. The single CSF biomarker $A \beta_{1-42}$ value showed the best diagnostic accuracy among the CSF biomarkers but the combined p-tau ${ }_{181} / \mathrm{A} \beta_{1-42}$ model improved numerically the specificity for the discrimination between $\mathrm{AD}$ dementia and SCD or depression.

It should be noted that monocentric studies might warrant biases, related to the great homogeneity of neuropsychological and biological assessments that would not allow for a generalization to more heterogeneous routine assessments in national or international patient populations. Thus, homogenization of standardized cognitive testing and procedures of biomarker analyses represent an important challenge for the international community. One should also note that the mean age at symptom onset of the participants of this investigation (64.1 \pm 9.3 years) is also lower than the mean age of AD patients diagnosed in France [43], which reflects our center's referral nature for young onset $\mathrm{AD}$ and rare dementias. This discrepancy, however, appears to be a methodological advantage because at younger ages, the symptoms are likely to be disease specific and not the manifestation of comorbidity or mixed pathologies that hinder the discriminative power of cognitive tests such as the FCSRT.

In conclusion, the FCSRT, which was already recommended for $\mathrm{AD}$ enrichment during the inclusion in clinical trials on SCD or MCI populations and which was thought to be useful to distinguish typical AD from other neurodegenerative conditions, has now demonstrated its value and discriminative reliability at the cohort level. The FCSRT should also be implemented in memory center's clinical routine keeping in mind that its specificity for typical AD is not absolute. However, decreased performance notably in delayed total recall of the FCSRT and intrusions were found to be particularly indicative of typical AD. Finally, our study emphasizes the existence of multiple "atypical AD" variants, among which PCA and logopenic primary progressive aphasia are the most prevalent, followed by CBS, nfv-PPA, sv-PPA, and bv-FTD.

\section{Acknowledgments}

This work was supported by funding from the program "Investissements d'avenir" ANR-10-IAIHU-06 (Agence Nationale de la Recherche-10-IA Agence Institut Hospitalo-Universitaire-6). HH is supported by the AXA Research Fund, the Fondation Université Pierre et Marie Curie, and the Fondation pour la Recherche sur Alzheimer, Paris, France. 


\section{RESEARCH IN CONTEXT}

1. Systematic review: We performed an exhaustive review of peer-reviewed articles (PubMed and MEDLINE) revealing not any investigation of the specificity for typical Alzheimer's disease (AD) of the Free and Cued Selective Reminding Test (FCSRT), recommended by the International Working Group.

2. Interpretation: Our clinical-cerebrospinal fluid (CSF) biomarker investigation of a large cohort of typical $\mathrm{AD}$ and eight other neurodegenerative diseases, subjective cognitive decline, and depression showed (1) the FCSRT sensitively detects typical AD in large patient settings with neurodegenerative diseases, (2) the FCRST has no absolute specificity at the individual level, (3) the spectrum of atypical AD should be opened up to multiple biomarker-positive degenerative diseases including, according to decreasing prevalence, posterior cortical atrophy, logopenic primary progressive aphasia, corticobasal syndrome, nonfluent/agrammatic primary progressive aphasia, semantic primary progressive aphasia, and frontotemporal dementia of the behavioral type.

3. Future directions: FCSRT reliability at the group level will be important for pharmacological trials by minimizing false non-AD inclusions. Widening the concept of atypical $\mathrm{AD}$ requires investigations of neuropathologic series to assess the proportions of $\mathrm{AD}$ in neurodegenerative diseases.

\section{References}

[1] Reitz C, Brayne C, Mayeux R. Epidemiology of Alzheimer disease. Nat Rev Neurol 2011;7:137-52.

[2] Dubois B, Feldman HH, Jacova C, Dekosky ST, Barberger-Gateau P, Cummings J, et al. Research criteria for the diagnosis of Alzheimer's disease: revising the NINCDS-ADRDA criteria. Lancet Neurol 2007; 6:734-46.

[3] Dubois B, Feldman HH, Jacova C, Hampel H, Molinuevo JL, Blennow K, et al. Advancing research diagnostic criteria for Alzheimer's disease: the IWG-2 criteria. Lancet Neurol 2014;13:614-29.

[4] Auriacombe S, Helmer C, Amieva H, Berr C, Dubois B, Dartigues JF. Validity of the free and cued selective reminding test in predicting dementia: the 3C study. Neurology 2010;74:1760-7.

[5] Epelbaum S, Benisty S, Reyes S, O’Sullivan M, Jouvent E, Düring M, et al. Verbal memory impairment in subcortical ischemic vascular disease: a descriptive analysis in CADASIL. Neurobiol Aging 2011; 32:2172-82.

[6] Grober E, Buschke H, Crystal H, Bang S, Dresner R. Screening for dementia by memory testing. Neurology 1988;38:900-3.

[7] Pasquier F, Grymonprez L, Lebert F, Van der Linden M. Memory impairment differs in frontotemporal dementia and Alzheimer's disease. Neurocase 2001;7:161-71.
[8] Pillon B, Deweer B, Agid Y, Dubois B. Explicit memory in Alzheimer's, Huntington's, and Parkinson's diseases. Arch Neurol 1993; 50:374-9.

[9] Sarazin M, Berr C, De Rotrou J, Fabrigoule C, Pasquier F, Legrain S, et al. Amnestic syndrome of the medial temporal type identifies prodromal AD: a longitudinal study. Neurology 2007;69:1859-67.

[10] Tounsi H, Deweer B, Ergis AM, Van der Linden M, Pillon B, Michon A, et al. Sensitivity to semantic cuing: an index of episodic memory dysfunction in early Alzheimer disease. Alzheimer Dis Assoc Disord 1999;13:38-46.

[11] Dubois B, Albert ML. Amnestic MCI or prodromal Alzheimer's disease? Lancet Neurol 2004;3:246-8.

[12] Jessen F, Amariglio RE, van Boxtel M, Breteler M, Ceccaldi M, Chételat G, et al. A conceptual framework for research on subjective cognitive decline in preclinical Alzheimer's disease. Alzheimers Dement 2014;10:844-52.

[13] Autorité de Santé H. Maladie d'Alzheimer: diagnostic et prise en charge. Saint-Denis La Plaine, France: Haute Autorité de Santé; 2011.

[14] Folstein MF, Folstein SE, McHugh PR. "Mini-mental state." A practical method for grading the cognitive state of patients for the clinician. J Psychiatr Res 1975;12:189-98.

[15] Dubois B, Slachevsky A, Litvan I, Pillon B. The FAB: a Frontal Assessment Battery at bedside. Neurology 2000;55:1621-6.

[16] Van der Linden M, Coyette F, Poitrenaud J, Kalafat M, Calicis F, Wyns C, et al. L'épreuve de rappel libre/rappel indicé à 16 items (RL/RI-16). In: Solal, ed. L'évaluation des troubles de la mémoire: présentation de quatre tests de mémoire épisodique avec leur étalonnage. France, Solal: Marseille; 2004. p. 25-47.

[17] Rascovsky K, Hodges JR, Knopman D, Mendez MF, Kramer JH, Neuhaus J, et al. Sensitivity of revised diagnostic criteria for the behavioural variant of frontotemporal dementia. Brain 2011;134:2456-77.

[18] Gorno-Tempini ML, Hillis AE, Weintraub S, Kertesz A, Mendez M, Cappa SF, et al. Classification of primary progressive aphasia and its variants. Neurology 2011;76:1006-14.

[19] Armstrong MJ, Litvan I, Lang AE, Bak TH, Bhatia KP, Borroni B, et al. Criteria for the diagnosis of corticobasal degeneration. Neurology 2013;80:496-503.

[20] Litvan I, Agid Y, Calne D, Campbell G, Dubois B, Duvoisin RC, et al. Clinical research criteria for the diagnosis of progressive supranuclear palsy (Steele-Richardson-Olszewski syndrome): report of the NINDSSPSP international workshop. Neurology 1996;47:1-9.

[21] Tang-Wai DF, Graff-Radford NR, Boeve BF, Dickson DW, Parisi JE, Crook R, et al. Clinical, genetic, and neuropathologic characteristics of posterior cortical atrophy. Neurology 2004;63:1168-74.

[22] McKeith IG, Dickson DW, Lowe J, Emre M, O’Brien JT, Feldman H, et al. Diagnosis and management of dementia with Lewy bodies: third report of the DLB Consortium. Neurology 2005;65:1863-72.

[23] American Psychiatric Association. Diagnostic and Statistical Manual of Mental Disorders, 5th Edition. Washington, DV: American Psychiatric Association; 2013.

[24] Montgomery SA, Asberg M. A new depression scale designed to be sensitive to change. Br J Psychiatry 1979;134:382-9.

[25] Fazekas F, Schmidt R, Kleinert R, Kapeller P, Roob G, Flooh E. The spectrum of age-associated brain abnormalities: their measurement and histopathological correlates. J Neural Transm Suppl 1998; 53:31-9.

[26] Caroppo P, Le Ber I, Camuzat A, Clot F, Naccache L, Lamari F, et al. Extensive white matter involvement in patients with frontotemporal lobar degeneration: think progranulin. JAMA Neurol 2014;71:1562-6.

[27] Seeburger JL, Holder DJ, Combrinck M, Joachim C, Laterza O, Tanen M, et al. Cerebrospinal fluid biomarkers distinguish postmortem-confirmed Alzheimer's disease from other dementias and healthy controls in the OPTIMA cohort. J Alzheimers Dis 2015;44:525-39.

[28] Tapiola T, Alafuzoff I, Herukka SK, Parkkinen L, Hartikainen P, Soininen H, et al. Cerebrospinal fluid \{beta\}-amyloid 42 and tau proteins as biomarkers of Alzheimer-type pathologic changes in the brain. Arch Neurol 2009;66:382-9. 
[29] Duits FH, Teunissen CE, Bouwman FH, Visser PJ, Mattsson N, Zetterberg H, et al. The cerebrospinal fluid "Alzheimer profile": easily said, but what does it mean? Alzheimers Dement 2014;10:713-23.

[30] McKhann GM, Knopman DS, Chertkow H, Hyman BT, Jack CR Jr, Kawas CH, et al. The diagnosis of dementia due to Alzheimer's disease: recommendations from the National Institute on Aging-Alzheimer's Association workgroups on diagnostic guidelines for Alzheimer's disease. Alzheimers Dement 2011;7:263-9.

[31] Di Stefano F, Epelbaum S, Coley N, Cantet C, Ousset PJ, Hampel H, et al. Prediction of Alzheimer's disease dementia: data from the GuidAge Prevention Trial. J Alzheimers Dis 2015;48:793-804.

[32] Wagner M, Wolf S, Reischies FM, Daerr M, Wolfsgruber S, Jessen F, et al. Biomarker validation of a cued recall memory deficit in prodromal Alzheimer disease. Neurology 2012;78:379-86.

[33] Desgranges B, Baron JC, Giffard B, Chételat G, Lalevée C, Viader F, et al. The neural basis of intrusions in free recall and cued recall: a PET study in Alzheimer's disease. Neuroimage 2002;17:1658-64.

[34] Cordato NJ, Halliday GM, Harding AJ, Hely MA, Morris JG. Regional brain atrophy in progressive supranuclear palsy and Lewy body disease. Ann Neurol 2000;47:718-28.

[35] Padovani A, Borroni B, Brambati SM, Agosti C, Broli M, Alonso R, et al. Diffusion tensor imaging and voxel based morphometry study in early progressive supranuclear palsy. J Neurol Neurosurg Psychiatry 2006;77:457-63.

[36] Hornberger M, Wong S, Tan R, Irish M, Piguet O, Kril J, et al. In vivo and post-mortem memory circuit integrity in frontotemporal dementia and Alzheimer's disease. Brain 2012;135:3015-25.
[37] Gorno-Tempini ML, Dronkers NF, Rankin KP, Ogar JM, Phengrasamy L, Rosen HJ, et al. Cognition and anatomy in three variants of primary progressive aphasia. Ann Neurol 2004;55:335-46.

[38] Lee GP, Loring DW, Flanigin HF, Smith JR, Meador KJ. Electrical stimulation of the human hippocampus produces verbal intrusions during memory testing. Neuropsychologia 1988;26:623-7.

[39] Slaets S, Le Bastard N, Theuns J, Sleegers K, Verstraeten A, De Leenheir E, et al. Amyloid pathology influences abeta1-42 cerebrospinal fluid levels in dementia with Lewy bodies. J Alzheimers Dis 2013; 35:137-46.

[40] Bouwman FH, van der Flier WM, Schoonenboom NS, van Elk EJ, Kok A, Rijmen F, et al. Longitudinal changes of CSF biomarkers in memory clinic patients. Neurology 2007;69:1006-11.

[41] Mesulam MM, Weintraub S, Rogalski EJ, Wieneke C, Geula C, Bigio EH. Asymmetry and heterogeneity of Alzheimer's and frontotemporal pathology in primary progressive aphasia. Brain 2014; 137:1176-92.

[42] Ewers M, Mattsson N, Minthon L, Molinuevo JL, Antonell A, Popp J, et al. CSF biomarkers for the differential diagnosis of Alzheimer's disease: a large-scale international multicenter study. Alzheimers Dement 2015;11:1306-15.

[43] Pradier C, Sakarovitch C, Le Duff F, Layese R, Metelkina A, Anthony S, et al. The mini mental state examination at the time of Alzheimer's disease and related disorders diagnosis, according to age, education, gender and place of residence: a cross-sectional study among the French National Alzheimer database. PLoS One 2014; 9:e103630. 